\title{
Infrared Matrix-Assisted Laser Desorption Electrospray Ionization (IR-MALDESI) Imaging Source Coupled to a FT-ICR Mass Spectrometer
}

\author{
Guillaume Robichaud, ${ }^{1}$ Jeremy A. Barry, ${ }^{1}$ Kenneth P. Garrard, ${ }^{2}$ David C. Muddiman ${ }^{1}$ \\ ${ }^{1}$ W. M. Keck FT-ICR Mass Spectrometry Laboratory, Department of Chemistry, North Carolina State University, Raleigh, \\ $\mathrm{NC}$, USA \\ ${ }^{2}$ Precision Engineering Center, North Carolina State University, Raleigh, NC, USA
}

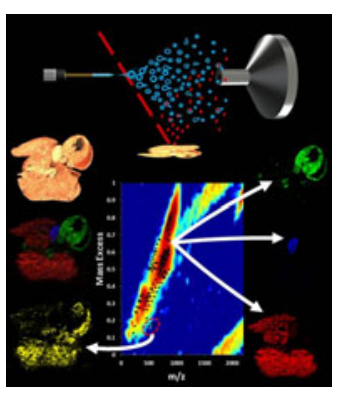

Abstract. Mass spectrometry imaging (MSI) allows for the direct monitoring of the abundance and spatial distribution of chemical compounds over the surface of a tissue sample. This technology has opened the field of mass spectrometry to numerous innovative applications over the past 15 years. First used with SIMS and MALDI MS that operate under vacuum, interest has grown for mass spectrometry ionization sources that allow for effective imaging but where the analysis can be performed at ambient pressure with minimal or no sample preparation. We introduce here a versatile source for MALDESI imaging analysis coupled to a hybrid LTQ-FT-ICR mass spectrometer. The imaging source offers single shot or multi-shot capability per pixel with full control over the laser repetition rate and mass spectrometer scanning cycle. Scanning rates can be as fast as $1 \mathrm{pixel} / \mathrm{second}$ and a spatial resolution of $45 \mu \mathrm{m}$ was achieved with oversampling.

Key words: FTMS, Mass spectrometry imaging, Infrared laser, MALDESI, Atmospheric pressure

Received: 14 August 2012/Revised: 14 September 2012/Accepted: 16 September 2012/Published online: 4 December 2012

\section{Introduction}

$\mathrm{T}$ he introduction of soft ionization sources such as matrix assisted laser desorption/ionization (MALDI) $[1,2]$ and electrospray ionization (ESI) [3] arguably revolutionized the field of mass spectrometry by allowing the analysis of large biomolecules. Another more recent development in mass spectrometry is the introduction of mass spectrometry imaging (MSI) in which material is desorbed from the sample surface, ionized and analyzed by a mass spectrometer while recording the spatial location. Using this additional layer of spatial information, heat maps are generated revealing the abundance and distribution of various compounds (e.g., peptides, drugs, metabolites, lipids, intact proteins) over the tissue area. Secondary ion mass spectrometry (SIMS) and MALDI MS were the first techniques to emerge in the field of mass spectrometry imaging [4-7] for biological applications. SIMS desorption with a focused ion beam can be performed with no sample

Electronic supplementary material The online version of this article (doi:10.1007/s13361-012-0505-9) contains supplementary material, which is available to authorized users.

Correspondence to: David Muddiman; e-mail: david_muddiman@ncsu.edu preparation allowing for nanometer resolution; however, its application range remains limited to smaller molecules. MALDI MS imaging routinely achieves soft ionization and mapping of biomolecules $>50 \mathrm{kDa}$ [8]. Spatial resolution, which is mainly defined by the laser spot size in MALDI imaging, can range from $100 \mu \mathrm{m}$ to as low as a few micrometers diameter when using a UV laser source. However, the trade-off between sensitivity and resolution has to be considered as the diameter is reduced [9]. On the down side, MALDI does require the application of an exogenous matrix and operates in vacuum which limits the type of sample that can be analyzed. Analysis is also limited to singly or doubly charged ions. Nevertheless, MALDI MS imaging remains the most commonly used imaging technique and a plethora of standalone sources or instruments that have incorporated MALDI imaging are now commercially available.

Due to the discrete nature of ion generation in MALDI and the fact that mainly singly-charged ions are produced, MALDI imaging sources have naturally been coupled to time-of-flight (TOF) instruments. However, due to the growing interest toward MSI of biological tissues, proteins and polypeptide identification, etc., many groups have turned toward instruments with higher resolving power and/or instruments with $\mathrm{MS}^{n}$ capability such as FT-ICR [10-12]. 
A trend among other ionization sources is to allow detection of multi-charged ions in their native environment, at ambient pressure and without sample preparation [13]. Among those, desorption electrospray ionization (DESI) [14] has also shown great imaging capabilities [15], recently achieving a spatial resolution of $35 \mu \mathrm{m}$ imaging brain tissue [16]. Another family of ambient ionization techniques have been developed and share the same working principle (i.e., the laser desorption of sample material with electrospray post-ionization [17-21]. The later techniques differ by their geometry or specific parameter setting (laser wavelength, fluence, etc.). Laser ablation electrospray ionization (LAESI) [22], which uses $2.94 \mu \mathrm{m}$ IR wavelength to ablate material from water rich samples, is to date the only one of these technique for which mass spectrometry imaging has been demonstrated [23-25]. In MALDESI, it is proposed that a laser (UV or IR) is used to excite the sample matrix (endogenous or exogenous) thereby facilitating the desorption of the analyte (liquid or solid) [2629]. We present here in detail the development of a versatile IR-MALDESI imaging source. The source is currently coupled to a commercial LTQ FT-ICR mass spectrometer and offers single shot or multi-shot capability per pixel with full control over the repetition rate and delay between ablation and the mass spectrometer trapping event through a user friendly Matlab interface. The source is equipped with a large enclosure that can be purged with nitrogen for precise control of relative humidity and abundance of ambient ions. A water cooled Peltier is used in lieu of a sample plate to control sample temperature and ice formation. Construction of the IRMALDESI ion source is presented in great detail, outlining the main design considerations. Effect of ablation/scan delay adjustment and injection time duration on abundance is shown using sections of dog liver tissue. The ability of the MALDESI source to resolve $45 \mu \mathrm{m}$ features by applying oversampling techniques is demonstrated and finally results of imaging experiments on mouse heart and lung tissue are presented.

\section{Experimental}

The MALDESI imaging source was developed from the current MALDESI source to perform mass spectrometry imaging and study the fundamentals of the MALDESI ionization process. The imaging source, shown in Figure 1, consists of a custom-built water cooled Peltier cold plate bolted on a vertical translation stage (NT66-508; Edmunds Optics, Barrington, NJ, USA) and placed on a XY motion controlled stage (LTA-HS; Newport Corporation, Irvine, CA, USA). Using a graphical user interface (GUI) developed with Matlab (MathWorks, Natick, MA, USA), the user can easily perform the system calibration, select the region of interest to be imaged on the sample, select imaging parameters, and finally start the imaging sequence. The source is fully enclosed and can be purged with nitrogen gas to control the relative humidity and ambient ion signal. Different components of the source are described in detail below.

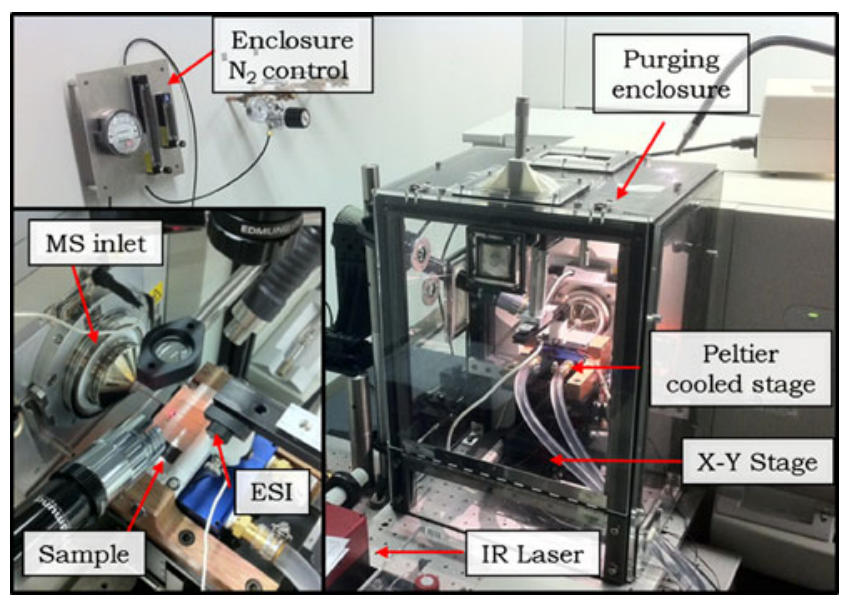

Figure 1. IR-MALDESI imaging source. ESI capillary, mass spectrometer inlet, and water cooled Peltier cold plate (bottom left) are located inside the large, air tight enclosure designed to control relative humidity and ambient ions while minimizing purging gas interaction with MALDESI process

\section{Materials}

HPLC grade acetonitrile and water were purchased from Burdick and Jackson (Musketon, MI, USA). Formic acid was purchased from Sigma Aldrich (St. Louis, MO, USA). Nitrogen (99.98\% purity) used to purge the MALDESI imaging source enclosure was obtained from MWSC High Purity Gases (Raleigh, NC, USA).

Mouse heart, lung and brain tissue samples were obtained from the NCSU College of Veterinary Medicine. Animals were housed and used in compliance with Institutional Animal Care and Use Committee and North Carolina State University regulations. The heart and lung tissues were cooled on dry ice subsequent to removal from the animal and stored at $-80{ }^{\circ} \mathrm{C}$ until sectioning. The brain tissue was snap-frozen in liquid nitrogen and stored at $-80{ }^{\circ} \mathrm{C}$. Fifty $\mu \mathrm{m}$ tissue slices were prepared using a CM1950 Cryostat (Leica, Buffalo Grove, IL, USA), placed on a microscope slide and analyzed without additional pretreatment. Fifty $\mu \mathrm{m}$-thick liver sections from a dog dosed with Tykerb were obtained from GlaxoSmithKline (Research Triangle Park, NC, USA). Calibration grids (G50-Ni and G100-Ni) were purchased from Electron Microscopy Science (Hatfield, PA, USA). For all tissue imaging experiments, stage temperature was maintained at $-8^{\circ} \mathrm{C}$. Each tissue slice or sample was placed on the stage in ambient air until a thin layer of frost appeared. The enclosure was then closed and nitrogen flow set manually to maintain a relative humidity of $10 \% \pm 2 \%$ and maintain frost layer thickness. Relative humidity was monitored using a temperature/relative humidity sensor (RHT20; Extech Instruments, Waltham, MA, USA).

\section{Mass Spectrometer}

The IR-MALDESI imaging source is coupled to a $7 \mathrm{~T}$ Thermo LTQ-FT-ICR (Thermo Fisher Scientific, San Jose, 
CA, USA). Tube lens voltage was set at $110 \mathrm{~V}$ and resolving power of the FT-ICR was set to $100,000_{\mathrm{FWHM}}$ at $\mathrm{m} / \mathrm{z} 400$. Automatic gain control (AGC) was turned off for the experiments. AGC is a prescan performed using the LTQ detector to individually set the injection time of each scan to regulate the number of ions reaching the FT-ICR and maintain mass measurement accuracy (MMA). Since the analyte ions in MALDESI enter the mass spectrometer as discrete packets (few milliseconds per ablation event) rather than being injected continuously, AGC is not applicable. Injection time was set at $150-200 \mathrm{~ms}$ for the present experiments and MMA remained within the instrument specifications. Adjustment of optimal injection time for our experiments will be further discussed in the result section.

An extended capillary was used to give adequate clearance for the imaging stage to raster the entire sampling area. The protruding tip was $23 \mathrm{~mm}$ long with a nominal ID of $0.596 \mathrm{~mm}$ so that conductance and pressure of the first pumping stage of the instrument remained unchanged when compared to the original instrument capillary. The length of the extended capillary was minimized to reduce the signal loss caused by the additional residence time of the ions in the capillary [30]. The temperature and voltage of the inlet capillary were respectively set at $250^{\circ} \mathrm{C}$ and $+37 \mathrm{~V}$.

\section{Laser and Optics}

The MALDESI source uses a wavelength tunable (2.7$3.1 \mu \mathrm{m}$ ) OPO laser with a pulse width of $7 \mathrm{~ns}$ (IR Opolette; Opotek, Carlsbad, CA, USA). Wavelength was set at $2.94 \mu \mathrm{m}(\mathrm{O}-\mathrm{H}$ stretch). Ablation energy at the target location was measured at $0.9 \mathrm{~mJ} /$ pulse. The laser beam was steered to the sampling plate using three metallic silver mirrors (Newport Corporation, Irvine, CA, USA) and focused to a diameter of $\sim 250 \mu \mathrm{m}$ with an uncoated $\mathrm{CaF}_{2}$ plano-convex lens (Edmund Optics, Barrington, NJ, USA) for an average surface power density of $2.6 \times 10^{12} \mathrm{Wm}^{-2}$. When multiple ablations per pixel were used during imaging experiments, the repetition rate was set at $20 \mathrm{~Hz}$.

\section{IR-MALDESI Source}

The electrospray solution was prepared by mixing a $1: 1$ solution ( $\mathrm{vol} / \mathrm{vol})$ of acetonitrile and water with $0.1 \%$ of formic acid. ESI solvent was electrosprayed at a flow rate of $1.4 \mu \mathrm{L} / \mathrm{min}$ through a $75 \mu \mathrm{m}$ i.d. fused silica emitter tapered to $30 \mu \mathrm{m}$ (New Objective, Woburn, MA, USA). The ESI voltage was tuned until a stable electrospray was obtained (circa $2.4 \mathrm{kV}$ ).

Optimized geometry parameters for the MALDESI imaging source were determined during a previous design of experiments [26]. Axial distance between the ESI tip and the mass spectrometer inlet was set at $9 \mathrm{~mm}$. IR laser was focused normal to the sample surface and distance between laser spot and mass spectrometer inlet was set at $8 \mathrm{~mm}$. Finally, the elevation difference between the sample surface and the mass spectrometer inlet and ESI capillary centerline was maintained at $5 \mathrm{~mm}$.

\section{Scanning Operation}

One important design objective for the MALDESI imaging source was to have full control over the number of laser shots per pixel, the frequency of laser shots, and also the delay between the laser ablation and the beginning of the mass spectrometer scan cycle. By controlling the scan/ ablation delay, one can adjust ion injection time duration to match the time lapse during which ablated material is entering the instrument, thereby maximizing the signal-tonoise ratio and reducing the variation in signal intensity. Gated trapping in-cell accumulation (ICA) [31, 32] for the pulsed ablation events could be used to further reduce the amount of ambient ions introduced in the mass spectrometer instrument by preventing ambient ions from getting in the cell between ablation events when performing imaging in multi-shot mode. Repeatability was also improved by adjusting the number of shots per pixel to ablate all the way through the tissue section so that volume of material ablated at each pixel location remains constant.

One common approach often used in UV-MALDI mass spectrometry imaging to account for variation in ion abundance is to average multiple scans per pixel location. This approach is not suitable for IR-MALDESI imaging due the larger amount of tissue ablated per single shot compared to UV-MALDI. Performing scan averaging with a FT-ICR would also significantly increase the dwell time at each pixel and therefore the overall imaging time per tissue section. For these reasons, each pixel corresponds to a single acquisition mass spectrum.

Matlab was chosen for programming the MALDESI imaging operator interface (see snapshot in Figure S1). A real time image from a fixed overhead camera (Chameleon; Point Grey Research Inc., Richmond, BC, Canada) is imbedded in the interface. Using a high resolution $25 \mathrm{~mm}$ fixed focal length lens (Edmunds Optics, Barrington, NJ, USA), a field of view of $38 \times 28 \mathrm{~mm}$ with an optical resolution of $59 \mu \mathrm{m}$ can be reached at a working distance of $150 \mathrm{~mm}$ with negligible barrel distortion. The camera is offset by a known distance with the laser spot, allowing both the laser and the camera to be aimed normal to the sample plane. The offset between the laser spot location and the camera field of view can be recalibrated within minutes if needed (see Figure S2).

Through the user interface, the operator first selects scanning parameters such as spot spacing, repetition rate, number of shots per pixel, ablation/scan delay, etc. The user selects a rectangular region of interest (ROI) on the sample of any size and aspect ratio by drawing a box on the video image with the mouse. Once all scanning parameters have been selected, the raster program is generated and loaded on the stage controller (ESP 300; Newport Corporation, Irvine, CA, USA) using a serial communication port as shown in 
Figure 2a). Laser ablation parameters (delay, repetition rate, shot per pixel, etc.) are also sent via serial communication to the MALDESI imaging control box which contain a microcontroller unit (Arduino Uno, Ivrea, Italy) and a house built laser Q-Switch trigger mechanism (pictures and detailed schematic can be found in Figures S3 to S5). Even though it is hardware specific, code used for Arduino microcontroller and Matlab interface can also be downloaded with supplementary material (for information only). Prior to starting the imaging sequence, a custom Component Object Model (COM) application written in $\mathrm{C}++$ is launched to take over the triggering of the mass spectrometer scans and allow communication with the MALDESI imaging control box via the mass spectrometer peripheral ports and a USB data acquisition board (OM-USB-1408FS; Omega Engineering, Stamford, CT, USA). When the imaging sequence is started via the user interface, the scanning loop shown (dashed square in Figure 2a) is initiated and digital signals are used to synchronize the stage movements, laser ablation and mass spectrometer scans. Details of this communication loop are shown in Figure 2b). The dwell time per pixel of IRMALDESI imaging source varies from 1 to $1.9 \mathrm{~s} /$ pixel depending on the resolving power and the ion injection time.

\section{Sample Temperature and Atmospheric Control}

The sampling surface consists of an aluminum 6061 cold plate $(44 \times 80 \times 10 \mathrm{~mm})$, which can be temperature regulated between $-10{ }^{\circ} \mathrm{C}$ and $+80{ }^{\circ} \mathrm{C}$ by controlling the voltage applied to a $50 \times 25 \mathrm{~mm}$ thermoelectric module (TE Technology, Traverse City, MI, USA). The hot side (bottom) of the thermoelectric element is water cooled using an aluminum water block (Custom Thermoelectric Inc., Bishopville, MD, USA) connected to an Exos-2 LX pump/ cooling unit (Koolance, Auburn, WA, USA). Water cooling of the thermoelectric element was preferred over air convection (fans) since it allows a more compact design while avoiding interaction between cooling airflow and the MALDESI desorption process.

A large purging enclosure was designed around the MALDESI source to control the ambient environment while imaging is performed. The total volume of the enclosure is 49 liters $(28 \times 35 \times 50 \mathrm{~cm})$. The enclosure is built with $6 \mathrm{~mm}$ thick clear cast acrylic panels bolted on a $25 \mathrm{~mm}$ construction rail frame (Thorlabs, Newton, NJ, USA). Neoprene foam is used to seal the frame-board interface and $150 \mu \mathrm{m}$ thick latex film is used to seal openings left for cables, camera, cooling water hoses and micrometer knobs. The laser beam enters the enclosure through a $1 \mathrm{~mm}$ thick uncoated sapphire window (Edmunds Optics, Barrington, NJ, USA). The MALDESI source can be easily accessed through the hinged front panel. Protective shields isolate the workspace from the rest of the laboratory and people entering the workspace wear proper eye protection.

The enclosure can be purged in a few minutes and a small positive pressure $(\sim 30 \mathrm{~Pa})$ maintained with a gas flow less than $7 \mathrm{~L} / \mathrm{min}$. Using a large enclosure and keeping the purging gas flow very low is key to minimize purging gas circulation that could interact with the desorption process.

There are several reasons why a purging system was implemented:

Frost and condensation control: Relative humidity in our facility varies on a daily basis from $15 \%$ to $50 \%$. When the (a)

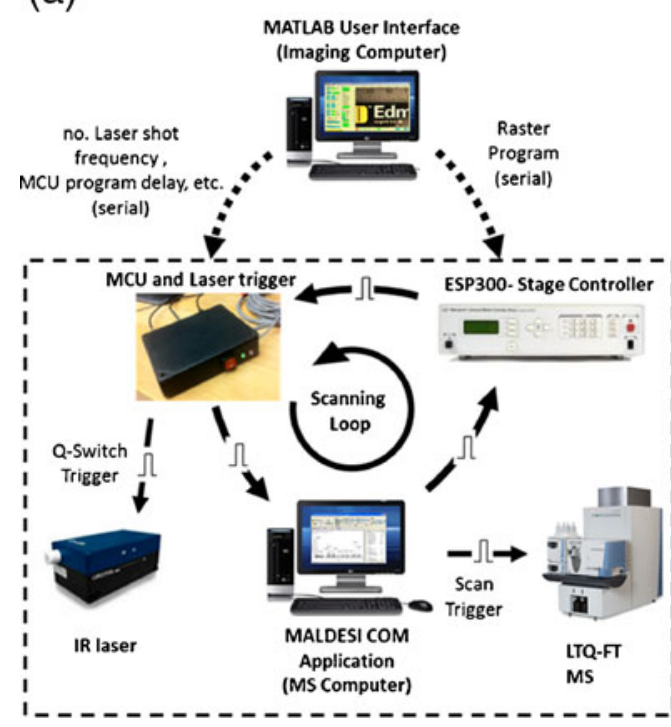

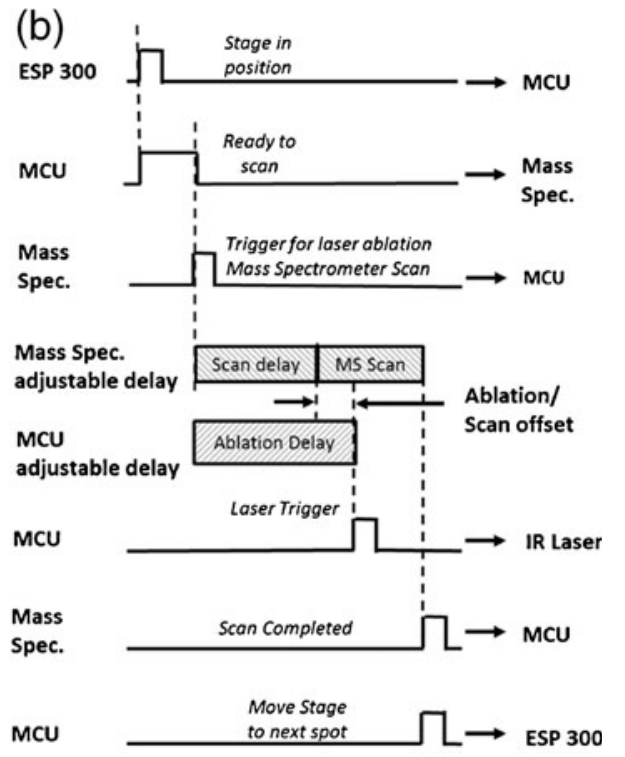

Figure 2. Communication between the different components of the MALDESI imaging source. (a) Scanning parameters are selected in the Matlab user interface and sent to the stage controller and the MALDESI controller box. Communication between components in dashed box (scanning loop) is then initiated. (b) Communication sequence for one pixel (one cycle of scanning loop) 
Peltier cooled stage is used to keep the tissue frozen during the imaging process, condensation and frost quickly build up on the surface of the sample (up to a few millimeters within few hours depending on ambient relative humidity). With this purging enclosure, $\mathrm{N}_{2}$ flow can be adjusted to prevent and/or control frost formation for applications where a thin layer of ice is used as a matrix [33, 34].

Eliminate ambient flow perturbation: One obvious advantage of performing MALDESI imaging in an enclosure is to eliminate any flow perturbation from building ventilation, entry of personnel in and out of the laboratory, etc.

Control ambient ions: Composition and intensity of ambient ions [35] can vary significantly from one day to another. The purging enclosure can be used to regulate the overall abundance of these ions during the MALDESI imaging.

\section{Data Processing and Analysis}

Datacube Explorer ver 0.7 (AMOLF) and Biomap (Novartis, Basel, Switzerland) were both considered as potential software to visualize the data collected by the MALDESI imaging system. Both applications are free [36] and have been used in multiple publications by different groups. However, Biomap could not be used since the dynamic range of ions collected with the FT-ICR was above the maximum value that the software can process (Biomap uses 16 bit signed integers to store abundance, which limits the maximum value to 32,767$)$. Datacube, on the other hand, is fast, relatively easy to use, and can handle large dynamic ranges. However, this fast processing comes at the price of a reduction in the $\mathrm{m} / \mathrm{z}$ scale resolution $(0.1 \mathrm{Da})$. This is an important limitation since the minimum increment between FT-ICR $m / z$ data points in a RAW file can be two to four orders of magnitude smaller. For these reasons and also to have full control over the processing of the mass spectrometer data, a custom MS imaging GUI was designed and programmed by our group (MSiReader) using Matlab (see Figure S6). The Bioinformatics, Statistics, and Image Processing toolboxes (MathWorks) are used by MSiReader.

The Thermo RAW file must first be converted to a .mzXML file using one of the free applications available online (e.g., ProteoWizard [37]). The .mzXML is loaded with MSiReader and converted to a structured array that contains all the scan information.

Listed below are a few of the additional features included in MSiReader:

Customizable heatmap appearance. Matlab's Colormap Editor can be used to customize heatmap appearance using an exhaustive list of tunable color scales. Different interpolation schemes can also be used to smooth contours of the heatmap (spline, linear, cubic, none). Order of the interpolation can be set from 0 to 5 . Images presented in this article were smoothed with a second order linear interpolation.
Mass spectrum extraction. Individual mass spectrum or averaged mass spectra of a user defined region of interest (ROI) can be generated. By defining two ROIs, a spectrum can be generated for all peaks in a region that are not in the other. The mass spectrum can be saved as a native Matlab .fig file for further analysis and formatting for presentation or publication.

Background subtraction. A file containing background noise spectra can be subtracted to the value presented on the heatmap. This feature can be used to eliminate electrospray and ambient ions from the output data.

Peak normalization. User has the option to normalize the abundance on the heatmap to the abundance of a specific $\mathrm{m} / \mathrm{z}$ value. For example, normalizing to the $\mathrm{m} / \mathrm{z}$ of an electrospray ion will reduce the abundance variation of the MALDESI signal that is caused by the variation in electrospray.

After submission of our work for publication, a recent publication on the visualization of high resolution FT-ICR imaging data with a new version of Data Cube was published and brought to our attention [38]; however, we did not utilize or have the opportunity to compare this software that was developed concurrently with ours. This will be the subject of a future study.

\section{Results and Discussion}

\section{Mass Spectrometer Scan Trigger Delay and Ion Injection Time}

A special COM application was created to calibrate the delay between the first laser pulse trigger and the beginning of the mass spectrometer scan cycle. Data was collected while rastering over a $50 \mu \mathrm{m}$ dog liver tissue section (monitoring homogeneous molecules). The delay value was incremented by $2 \mathrm{~ms}$ for each scan over a specified delay range. By setting the injection time to match the total ablation duration (\#pulses $\times 50 \mathrm{~ms}$ for $20 \mathrm{~Hz}$ laser repetition rate) the maximum abundance will occur when the LTQ trap opens as the ablated material enters the mass spectrometer. As seen in Figure 3a), the ion abundance is maximized when the laser ablation is triggered $15 \mathrm{~ms}$ after triggering the mass spectrometer scan sequence. Note that this delay is an adjustable variable within the MALDESI imaging system and accounts for any software or hardware communication overhead delay between the moment the mass spectrometer receives the trigger and the moment the ions are actually collected.

Once the optimal delay of $15 \mathrm{~ms}$ was set, the effect of the total ion injection time on abundance was determined. For these experiments, a series of 50 scans were collected with three laser pulses per scan and a specified ion injection time $(50,100,150,200,250,300,400$, or $500 \mathrm{~ms})$. As shown in Figure $3 \mathrm{~b}$ ), an initial increase in abundance from 50 to $150 \mathrm{~ms}$ is observed, whereas increasing the ion injection 
(a)

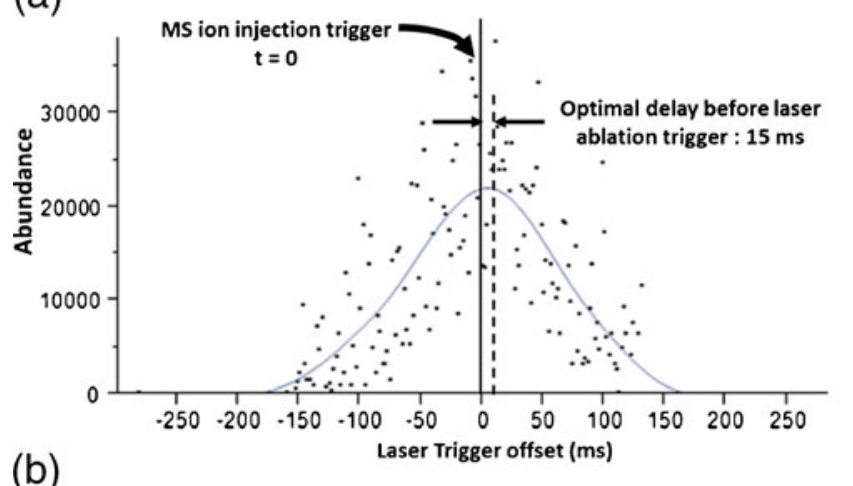

(b)

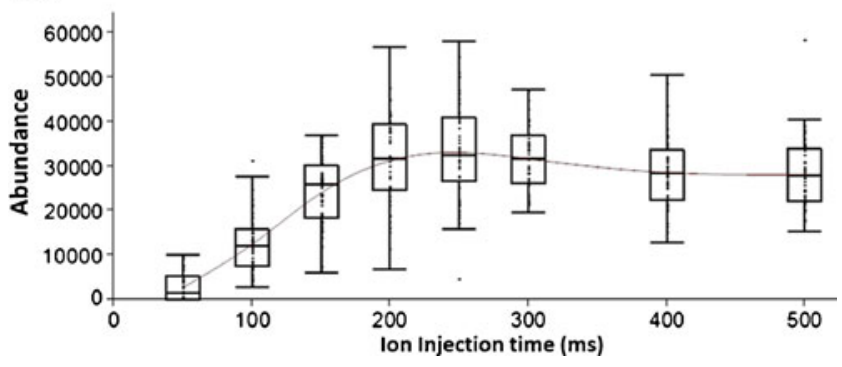

Figure 3. Sum of abundance of three lipid peaks from dog liver tissue $(758.57 \mathrm{Da} / 786.60 \mathrm{Da} / 810.60 \mathrm{Da})$ for three shots per pixel. (a) The effect of different delays between laser ablation and mass spectrometer scan triggers. (b) The effect of increasing the ion injection time on the abundance of these tissue ions

time past $150 \mathrm{~ms}$ does not result in significant signal loss. At $20 \mathrm{~Hz}$ repetition rate, each laser pulse takes roughly $50 \mathrm{~ms}$, so with an ion injection time of $50 \mathrm{~ms}$, only ions generated from the first pulse are allowed into the trap. With a $150 \mathrm{~ms}$ ion injection time, the ions generated from all three consecutive laser pulses are collected in the trap. The linear increase in abundance is, therefore, attributable to the fact that more material is ablated (three shots at $20 \mathrm{~Hz}$ ) and collected in the ion trap. Even though increasing the injection time has little effect on the abundance of the tissue ions, it does affect the total ion current (TIC) since more ambient ions are collected. With the AGC turned off this can adversely affect the MMA due to space-charge effects [39, 40]. Even though the AGC is turned off when performing MALDESI imaging, the Thermo LTQ-FT-ICR instrument will still use the calibration parameters (A and $\mathrm{B}$ parameters) corresponding to the specified AGC setting to calculate the mass-to-charge. During the set of experiments presented in Figure $3 b$, the total ion current increased as the injection time was raised since more ambient ions were allowed to enter the instrument. The MMA of ions of known mass (e.g., $m / z 369.3516$ cholesterol $\left(\mathrm{M}-\mathrm{H}_{2} \mathrm{O}+\mathrm{H}^{+}\right)^{+}$and $\mathrm{m} / \mathrm{z}$ 581.1420 Tykerb $\left(\mathrm{M}+\mathrm{H}^{+}\right)^{+}$were calculated for each spectrum measured at those different injection times. As expected, the observed mass of those ions drifts toward higher values as the TIC increases due to space-charge effects. However, for injection time values currently used with the IR-MALDESI imaging source (150-200 ms), the average MMA for those ions did not exceed $0.6 \mathrm{ppm}$, which remains well within instrument specifications.

\section{Imaging Resolution}

Both under sampling and oversampling imaging techniques [6] have been explored with IR-MALDESI imaging. For the under sampling method, the number of shots per pixel is adjusted to ablate through the tissue so the amount of material remains constant without overlapping. Even though the measured focus diameter of the IR beam is around $250 \mu \mathrm{m}$ as measured from the laser spot size on thermal paper, it is possible to obtain resolution smaller than $250 \mu \mathrm{m}$ with no or very little overlapping when performing tissue imaging. Indeed, considering a Gaussian laser beam distribution, the desorption focus diameter or the surface for which irradiance is higher than the tissue desorption threshold can be significantly smaller than the optical focus diameter [41]. The abundance of lipid peaks on a $50 \mu \mathrm{m} \mathrm{dog}$ liver tissue as a function of the number of shots is shown in Figure 4. It shows that after five shots, the abundance no longer increases, and that a maximum ablation diameter size of $150 \mu \mathrm{m}$ has been reached. The number of pulses necessary to reach that maximum value depends on the laser fluence, the tissue type, section thickness, and section condition (fresh, frozen, dry, etc.). It is, therefore, possible to perform IR-MALDESI imaging with smaller resolution than the measured focal spot size without any oversampling or overlapping. Oversampling techniques $[42,43]$ have also been explored to improve spatial resolution. When an oversampling method is used, the step size is significantly smaller than the actual desorption diameter, such that only a fraction of the spot size area will be ablated. Using this technique, features much smaller than the desorption diameter can be resolved. Electron microscope calibration grids of different sizes were used to evaluate the spatial resolution of the imaging technique. Rather than spotting the

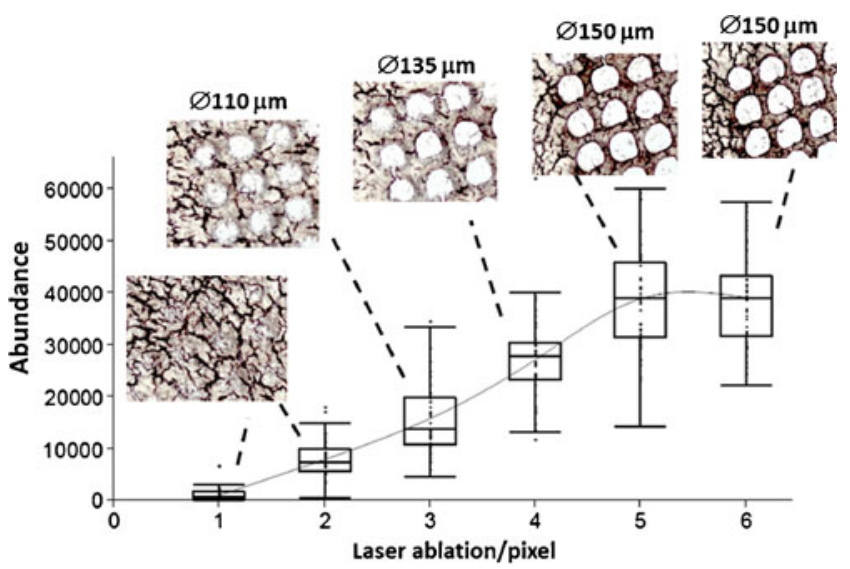

Figure 4. Signal magnitude and crater size as a function of the number of shot/pixel for injection time of $500 \mathrm{~ms}$. Signal intensity increases until a maximum crater size is obtained 
(a)

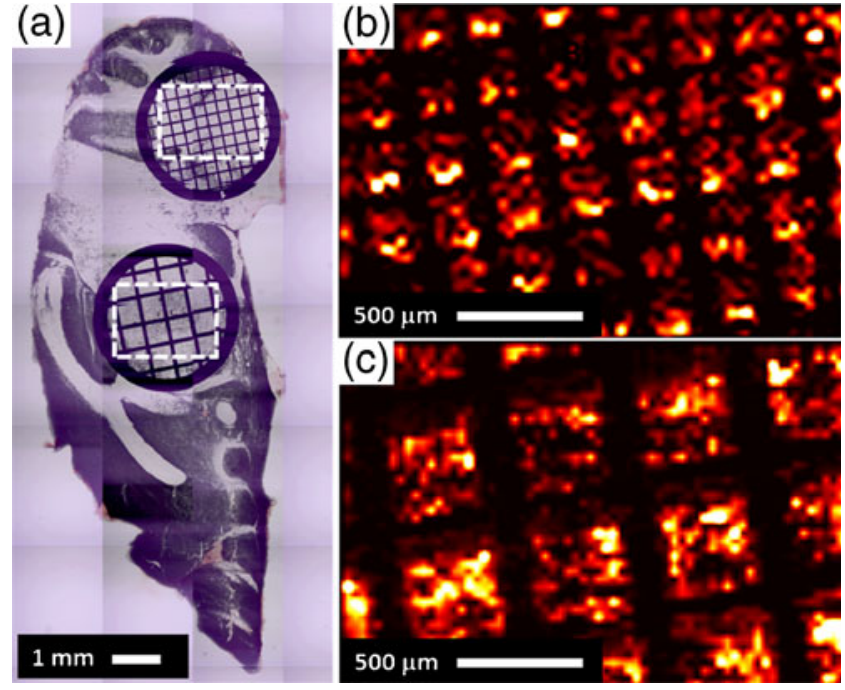

Figure 5. (a) Electron microscope calibration grid used to mask $50 \mu \mathrm{m}$ thick brain tissue and test resolution of MALDESI imaging source. Grid bar width as small as (b) $45 \mu \mathrm{m}$ and (c) $80 \mu \mathrm{m}$ were resolved using oversampling technique with a spot spacing of $30 \mu \mathrm{m}$. lon: $\mathrm{m} / \mathrm{z} 369.352$ cholesterol $\left[\mathrm{M}-\mathrm{H}_{2} \mathrm{O}+\mathrm{H}\right]+$ presented with a bin size of $0.01 \mathrm{Da}$

grid with a high concentration of a known analyte [42, 44], the grid was used as a mask [43] over a $50 \mu \mathrm{m}$ mouse brain tissue sample. Some authors have defined the resolution as the step size for which the intensity increases from $20 \%$ to $80 \%$ of its maximum value [43-46], which often leads to a smaller resolution claim. However, we prefer the more conservative definition of resolution as being the smallest feature that can be spatially resolved $[16,42]$. As shown in Figure 5, features as small as $45 \mu \mathrm{m}$ (width of electron microscope grid bars) were resolved using $30 \mu \mathrm{m}$ laser spot spacing, two shots/pixels, and injection time of $250 \mathrm{~ms}$.

\section{Tissue Imaging of Mouse Heart and Lungs}

Imaging results for a section of mouse heart and lung are presented in Figure 6. These images are presented with no background subtraction and $\mathrm{S} / \mathrm{N}$ varies from as low as 2-3:1 $(\mathrm{m} / \mathrm{z} 216.088)$ to as high as 200-300:1 ( $\mathrm{m} / \mathrm{z}$ 734.571). Acquisition of the 5108 scans was performed in $\sim 2.5 \mathrm{~h}$. Over 175 individual peaks that are coincident with tissue outline were found by browsing manually through the file using the imaging software. Some of these peaks corresponding to specific features such as the heart, lungs, blood, or fatty tissues are presented in Figure 6a. Mass excesses of these peaks, defined as the nominal mass subtracted from the monoisotopic mass [47] (sometime also defined as mass defects [48]), were compared with those of all entries from LIPID MAPS Structure Database (LDMS) [49, 50] (except those not likely to be found on those organs, such as free fatty acyls and polyketides [51, 52]). A lipid mass excess map was generated by plotting the mass excess as a function of the $m / z$ of all 25,000 entries from the LDMS. The lipid distribution was converted to a $\log _{2}$ probability map and is presented in Figure 6b). The pattern found in the lipid
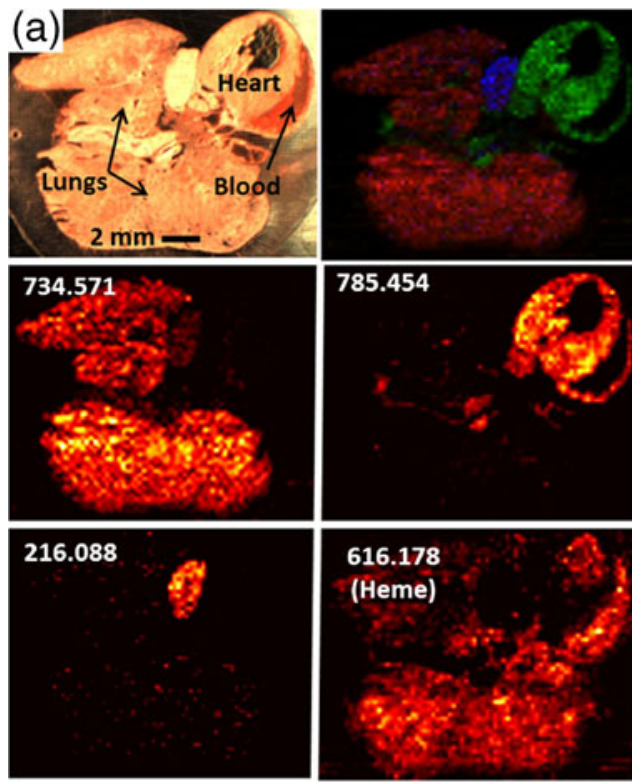
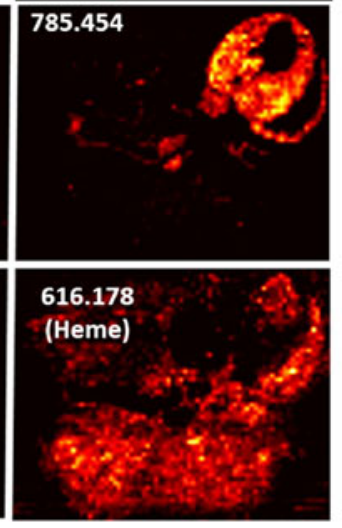

(b)

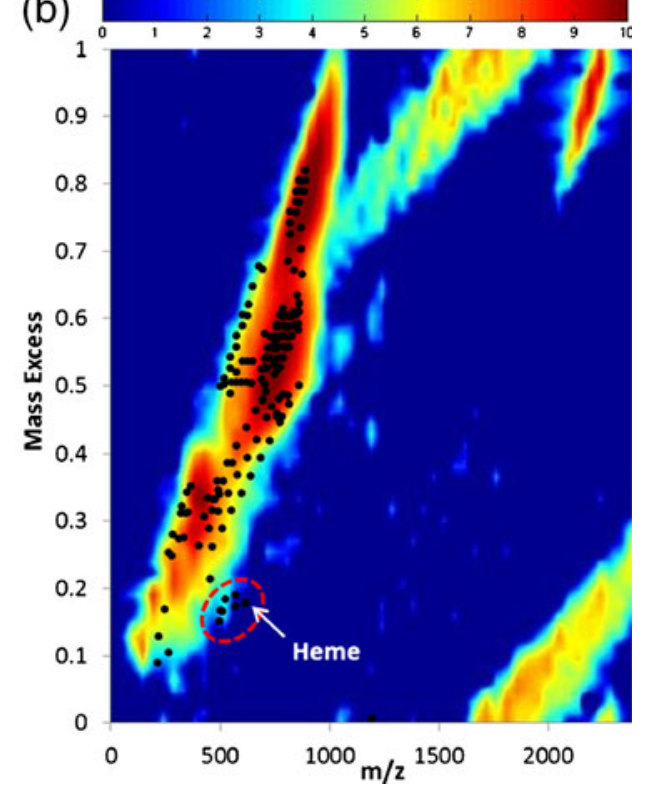

Figure 6. (a) Selection of peaks found on heart and lungs of adult mouse with different spatial distributions using three shots/ pixels and an injection time of $200 \mathrm{~ms}$ and colocalization map of some those peaks (top right). Bin size used was $0.01 \mathrm{Da}$ and values shown on each image is the $\mathrm{m} / \mathrm{z}$ center. (b) Mass excess of all tissue related peaks overlapped with mass excess distribution for lipids from LIPID MAPS Structure Database ( $\log _{2}$ scale). Location of peaks found on tissue suggests that most are lipids except for a few clusters for example the molecules sharing the Heme group spatial distribution $(\mathrm{m} / \mathrm{z} 616.178)$ or $\mathrm{m} / \mathrm{z}$ 216.088 
distribution is explained by the fact that some lipids share the same functional group but are characterized by the length of their chains, often composed of repeated units. Those lipid distributions will appear as a stretched cluster in the lipid mass excess space. It appears that most of the ions found on the tissue are lipids since they overlap with the lipid distribution except for some that fall in the lipid forbidden zone such as heme $\mathrm{b}[\mathrm{M}+\mathrm{H}]^{+}(\mathrm{m} / \mathrm{z}$ 616.178) and other ions sharing the same spatial distribution.

\section{Conclusion}

A versatile IR-MALDESI imaging source was designed and implemented for a commercial FT-ICR mass spectrometer. Spatial resolution of $45 \mu \mathrm{m}$ was demonstrated using oversampling techniques. Imaging can be performed with dwell time as low as $1 \mathrm{~s} /$ pixel in single or multi-shot mode with full control over the repetition rate and the delay between the ablation event and the mass spectrometer scan delay. This level of control can be used to improve signal repeatability and signal-to-noise ratio. Spatial resolution could also be further improved by optically reducing the laser beam spot size. Experimental space covered by fluence, tissue thickness, spatial resolution, etc. in IR-MALDESI imaging is currently being investigated.

\section{Acknowledgments}

The authors thank Dr. Michael W. Senko at ThermoFisher Scientific for providing the Component Object Model (COM) library for their instrument and Dr. Troy Ghashghaei from NCSU College of Veterinary Medicine for providing the mouse tissue samples. The authors thank Dr. Reid Groseclose, Dr. Stephen Castellino, and Dr. David Wagner from GSK for providing the dog liver samples. Finally, the authors gratefully acknowledge the financial support received from the National Institutes of Health (R01GM087964), the W. M. Keck Foundation, and North Carolina State University.

\section{References}

1. Karas, M., Hillenkamp, F.: Laser desorption ionization of proteins with molecular masses exceeding 10,000 daltons. Anal. Chem. 60(20), 22992301 (1988)

2. Tanaka, K., Waki, H., Ido, Y., Akita, S., Yoshida, Y., Yoshida, T., Matsuo, T.: Protein and polymer analysis up to $m / z 100,000$ by laser ionization time-of-flight mass spectrometry. Rapid Commun. Mass Spectrom. 2(8), 151-153 (1988)

3. Fenn, J.B., Mann, M., Meng, C.K., Wong, S.F., Whitehouse, C.M.: Electrospray ionization for mass spectrometry of large biomolecules. Science 246(4926), 64-71 (1989)

4. Caprioli, R.M., Farmer, T.B., Gile, J.: Molecular imaging of biological samples: localization of peptides and proteins using MALDI-TOF MS. Anal. Chem. 69(23), 4751-4760 (1997)

5. Todd, P.J., McMahon, J.M., Short, R.T., McCandlish, C.A.: Organic SIMS of biologic tissue. Anal. Chem. 69(17), 529A-535A (1997)

6. Todd, P.J., Schaaff, T.G., Chaurand, P., Caprioli, R.M.: Organic ion imaging of biological tissue with secondary ion mass spectrometry and matrix-assisted laser desorption/ionization. J. Mass Spectrom. 36(4), 355-369 (2001)

7. McDonnell, L.A., Heeren, R.M.: Imaging mass spectrometry. Mass Spectrom. Rev. 26(4), 606-643 (2007)

8. Chughtai, K., Heeren, R.M.: Mass spectrometric imaging for biomedical tissue analysis. Chem. Rev. 110(5), 3237-3277 (2010)

9. Chaurand, P., Schriver, K.E., Caprioli, R.M.: Instrument design and characterization for high resolution MALDI-MS imaging of tissue sections. J. Mass Spectrom. 42(4), 476-489 (2007)

10. Taban, I.M., Altelaar, A.F.M., Van der Burgt, Y.E.M., McDonnell, L.A., Heeren, R.M.A., Fuchser, J., Baykut, G.: Imaging of peptides in the rat brain using MALDI-FTICR mass spectrometry. J. Am. Soc. Mass Spectrom. 18(1), 145-151 (2007)

11. Cornett, D.S., Frappier, S.L., Caprioli, R.M.: MALDI-FTICR imaging mass spectrometry of drugs and metabolites in tissue. Anal. Chem. 80 (14), 5648-5653 (2008)

12. Pol, J., Vidova, V., Kruppa, G., Kobliha, V., Novak, P., Lemr, K., Kotiaho, T., Kostiainen, R., Havlicek, V., Volny, M.: Automated ambient desorption-ionization platform for surface imaging integrated with a commercial Fourier transform ion cyclotron resonance mass spectrometer. Anal. Chem. 81(20), 8479-8487 (2009)

13. Weston, D.J.: Ambient ionization mass spectrometry: current understanding of mechanistic theory; analytical performance, and application areas. Analyst 135(4), 661-668 (2010)

14. Takats, Z., Wiseman, J.M., Gologan, B., Cooks, R.G.: Mass spectrometry sampling under ambient conditions with desorption electrospray ionization. Science 306(5695), 471-473 (2004)

15. Wiseman, J.M., Puolitaival, S.M., Takats, Z., Cooks, R.G., Caprioli, R.M.: Mass spectrometric profiling of intact biological tissue by using desorption electrospray ionization Angew. Chem. Int. Ed. 44(43), 70947097 (2005)

16. Campbell, D.I., Ferreira, C.R., Eberlin, L.S., Cooks, R.G.: Improved spatial resolution in the imaging of biological tissue using desorption electrospray ionization. Anal. Bioanal. Chem. 404(2), 389-398 (2012)

17. Brady, J.J., Judge, E.J., Levis, R.J.: Mass spectrometry of intact neutral macromolecules using intense non-resonant femtosecond laser vaporization with electrospray post-ionization. Rapid Commun. Mass Spectrom. 23(19), 3151-3157 (2009)

18. Jorabchi, K., Smith, L.M.: Single droplet separations and surface partition coefficient measurements using laser ablation mass spectrometry. Anal. Chem. 81(23), 9682-9688 (2009)

19. Liu, J., Qiu, B., Luo, H.: Fingerprinting of yogurt products by laser desorption spray post-ionization mass spectrometry. Rapid Commun. Mass Spectrom. 24(9), 1365-1370 (2010)

20. Rezenom, Y.H., Dong, J., Murray, K.K.: Infrared laser-assisted desorption electrospray ionization mass spectrometry. Analyst 133(2), 226-232 (2008)

21. Shiea, J., Huang, M.Z., Hsu, H.J., Lee, C.Y., Yuan, C.H., Beech, I., Sunner, J.: Electrospray-assisted laser desorption/ionization mass spectrometry for direct ambient analysis of solids. Rapid Commun. Mass Spectrom. 19(24), 3701-3704 (2005)

22. Nemes, P., Vertes, A.: Laser ablation electrospray ionization for atmospheric pressure, in vivo, and imaging mass spectrometry. Anal. Chem. 79(21), 8098-8106 (2007)

23. Nemes, P., Barton, A.A., Li, Y., Vertes, A.: Ambient molecular imaging and depth profiling of live tissue by infrared laser ablation electrospray ionization mass spectrometry. Anal. Chem. 80(12), 4575-4582 (2008)

24. Nemes, P., Barton, A.A., Vertes, A.: Three-dimensional imaging of metabolites in tissues under ambient conditions by laser ablation electrospray ionization mass spectrometry. Anal. Chem. 81(16), 66686675 (2009)

25. Nemes, P., Woods, A.S., Vertes, A.: Simultaneous imaging of small metabolites and lipids in rat brain tissues at atmospheric pressure by laser ablation electrospray ionization mass spectrometry. Anal. Chem. 82(3), 982-988 (2010)

26. Barry, J.A., Muddiman, D.C.: Global optimization of the infrared matrix-assisted laser desorption electrospray ionization (IR MALDESI) source for mass spectrometry using statistical design of experiments Rapid Commun. Mass Spectrom. 25(23), 3527-3536 (2011)

27. Sampson, J.S., Hawkridge, A.M., Muddiman, D.C.: Generation and detection of multiply-charged peptides and proteins by matrix-assisted laser desorption electrospray ionization (MALDESI) Fourier transform ion cyclotron resonance mass spectrometry. J. Am. Soc. Mass Spectrom. 17(12), 1712-1716 (2006) 
28. Sampson, J.S., Hawkridge, A.M., Muddiman, D.C.: Construction of a versatile high precision ambient ionization source for direct analysis and imaging. J. Am. Soc. Mass Spectrom. 19(10), 1527-1534 (2008)

29. Sampson, J.S., Murray, K.K., Muddiman, D.C.: Intact and top-down characterization of biomolecules and direct analysis using infrared matrix-assisted laser desorption electrospray ionization coupled to FTICR mass spectrometry. J. Am. Soc. Mass Spectrom. 20(4), 667-673 (2009)

30. Lin, B.W., Sunner, J.: Ion-transport by viscous-gas flow-through capillaries. J. Am. Soc. Mass Spectrom. 5(10), 873-885 (1994)

31. Kutz, K.K., Schmidt, J.J., Li, L.: In situ tissue analysis of neuropeptides by MALDI FTMS in-cell accumulation. Anal. Chem. 76(19), 5630 5640 (2004)

32. O'Connor, P.B., Costello, C.E.: Application of multishot acquisition in Fourier transform mass spectrometry. Anal. Chem. 72(20), 5125-5130 (2000)

33. Berkenkamp, S., Karas, M., Hillenkamp, F.: Ice as a matrix for IRmatrix-assisted laser desorption/ionization: mass spectra from a protein single crystal. Proc. Natl. Acad. Sci. U. S. A. 93(14), 7003-7007 (1996)

34. Von Seggern, C.E., Gardner, B.D., Cotter, R.J.: Infrared atmospheric pressure MALDI ion trap mass spectrometry of frozen samples using a Peltier-cooled sample stage. Anal. Chem. 76(19), 5887-5893 (2004)

35. Keller, B.O., Sui, J., Young, A.B., Whittal, R.M.: Interferences and contaminants encountered in modern mass spectrometry. Anal. Chim. Acta 627(1), 71-81 (2008)

36. msimaging: http://www.maldi-msi.org/ Accessed

37. ProteoWizard: http://proteowizard.sourceforge.net/ Accessed

38. Smith, D.F., Kharchenko, A., Konijnenburg, M., Klinkert, I., PasaTolic, L., Heeren, R.M.: Advanced mass calibration and visualization for FT-ICR mass spectrometry imaging. J. Am. Soc. Mass Spectrom 23(11), 1865-1872 (2012)

39. Jeffries, J.B., Barlow, S.E., Dunn, G.H.: Theory of space-charge shift of ion-cyclotron resonance frequencies. Int. J. Mass Spectrom. Ion Process. 54(1/2), 169-187 (1983)

40. Francl, T.J., Sherman, M.G., Hunter, R.L., Locke, M.J., Bowers, W.D., Mciver, R.T.: Experimental-determination of the effects of space-charge on ion-cyclotron resonance frequencies. Int. J. Mass Spectrom. Ion Process. 54(1/2), 18-199 (1983)

41. Koestler, M., Kirsch, D., Hester, A., Leisner, A., Guenther, S., Spengler, B.: A high-resolution scanning microprobe matrix-assisted laser desorption/ionization ion source for imaging analysis on an ion trap/Fourier transform ion cyclotron resonance mass spectrometer. Rapid Commun. Mass Spectrom. 22(20), 3275-3285 (2008)

42. Jurchen, J.C., Rubakhin, S.S., Sweedler, J.V.: MALDI-MS imaging of features smaller than the size of the laser beam. J. Am. Soc. Mass Spectrom. 16(10), 1654-1659 (2005)

43. Li, Y., Shrestha, B., Vertes, A.: Atmospheric pressure molecular imaging by infrared MALDI mass spectrometry. Anal. Chem. 79(2), 523-532 (2007)

44. Luxembourg, S.L., Mize, T.H., McDonnell, L.A., Heeren, R.M.: Highspatial resolution mass spectrometric imaging of peptide and protein distributions on a surface. Anal. Chem. 76(18), 5339-5344 (2004)

45. Colliver, T.L., Brummel, C.L., Pacholski, M.L., Swanek, F.D., Ewing, A.G., Winograd, N.: Atomic and molecular imaging at the single-cell level with TOF-SIMS. Anal. Chem. 69(13), 2225-2231 (1997)

46. Luxembourg, S.L., McDonnell, L.A., Mize, T.H., Heeren, R.M.A.: XInfrared mass spectrometric imaging below the diffraction limit. $J$. Proteome Res. 4(3), 671-673 (2005)

47. Frahm, J.L., Howard, B.E., Heber, S., Muddiman, D.C.: Accessible proteomics space and its implications for peak capacity for zero-, one-, and two-dimensional separations coupled with FT-ICR and TOF mass spectrometry. J. Mass Spectrom. 41(3), 281-288 (2006)

48. Jones, J.J., Stump, M.J., Fleming, R.C., Lay Jr., J.O., Wilkins, C.L.: Strategies and data analysis techniques for lipid and phospholipid chemistry elucidation by intact cell MALDI-FTMS. J. Am. Soc. Mass Spectrom. 15(11), 1665-1674 (2004)

49. Sud, M., Fahy, E., Cotter, D., Brown, A., Dennis, E.A., Glass, C.K., Merrill Jr., A.H., Murphy, R.C., Raetz, C.R., Russell, D.W., Subramaniam, S.: LMSD: LIPID MAPS structure database. Nucleic Acids Res. 35(Database issue), D527-532 (2007)

50. LIPID MAPS Structure Database (LDMS): http://www.lipidmaps.org/ data/structure/index.html. Accessed

51. Christie, W.W.: Rapid separation and quantification of lipid classes by high performance liquid chromatography and mass (light-scattering) detection. J. Lipid Res. 26(4), 507-512 (1985)

52. Fahy, E., Subramaniam, S., Brown, H.A., Glass, C.K., Merrill Jr., A.H., Murphy, R.C., Raetz, C.R., Russell, D.W., Seyama, Y., Shaw, W., Shimizu, T., Spener, F., van Meer, G., VanNieuwenhze, M.S., White, S.H., Witztum, J.L., Dennis, E.A.: A comprehensive classification system for lipids. J. Lipid Res. 46(5), 839-861 (2005) 